\title{
On Biharmonic Lorentz Hypersurfaces with Non-Diagonal Shape Operator
}

\author{
Deepika and Ram Shankar Gupta*
}

(Communicated by Murat Tosun)

\begin{abstract}
We prove that there exist no proper biharmonic Lorentz hypersurface $M_{1}^{n}$ in $E_{1}^{n+1}$ with at most three distinct principal curvatures of non-diagonal shape operator having minimal polynomial $(y-\lambda)^{2}\left(y-\lambda_{1}\right)\left(y-\lambda_{n}\right)$.
\end{abstract}

Keywords: Pseudo-Euclidean space; Biharmonic submanifolds; Mean curvature vector.

AMS Subject Classification (2010): Primary: 53D12 ; Secondary: 53C40; 53C42.

\section{Introduction}

Let $M_{r}^{n}$ be an $n$-dimensional, connected submanifold of the pseudo-Euclidean space $E_{s}^{m}$. Denote by $\vec{x}$ and $\triangle$ respectively the position vector field and the Laplace operator on $M_{r}^{n}$ with respect to the induced metric $g$ on $M_{r}^{n}$, from the indefinite metric on the ambient space $E_{s}^{m}$. It is well known that

$$
\triangle \vec{x}=-n \vec{H}
$$

where $\vec{H}$ is the mean curvature vector of $M$. An immersion is minimal $(\vec{H}=0)$ if and only if $\triangle \vec{x}=0$ and is called biharmornic if $\triangle^{2} \vec{x}=0$ i.e. $\triangle \vec{H}=0$. Of course, for an immersion, minimality implies biharmonicity.

The study of submanifolds with harmonic mean curvature vector field was initiated by Chen in 1985 and arose in the context of his theory of submanifolds of finite type. A survey on submanifolds of finite type and various related topics was presented in $[4,5]$.

In 1991, Chen conjectured the following:

Conjecture: The only biharmonic submanifolds of Euclidean spaces are the minimal ones.

In Euclidean spaces, we have the following results, which indeed support the above mentioned conjecture. Chen proved in 1985 that every biharmonic surface in $E^{3}$ is minimal. Thereafter, I. Dimitric generalized this result [9]. In [14], it was proved that every biharmonic hypersurface in $E^{4}$ is minimal. In [16], it was obtained that every biharmonic hypersurface in $E^{5}$ with three distinct principal curvatures must be minimal. Also, it was proved that every biharmonic hypersurfaces with three distinct principal curvatures in $E^{n+1}$ with arbitrary dimension is minimal [12]. Recently, it was proved that there exist no proper biharmonic hypersurfaces in $E^{5}$ with zero scalar curvature [10].

Chen et al. $[7,8]$ obtained some examples of proper biharmonic surfaces in 4-dimensional pseudo-Euclidean spaces $E_{s}^{4}$ for $s=1,2,3$ (see also [6]). Also, it was proved in [7, 8] that biharmonic surfaces in pseudoEuclidean 3-spaces are minimal. A. Arvanitoyeorgos et al. [2] proved that biharmonic Lorentzian hypersurfaces in Minkowski 4-spaces are minimal. In [16], it was proved that every biharmonic non-degenerate hypersurface in $E_{s}^{5}$ with three distinct principal curvatures of diagonal shape operator is minimal.

In this paper, we study biharmonic Lorentz hypersurfaces $M_{1}^{n}$ in $E_{1}^{n+1}$ with at most three distinct eigen values of non-diagonal shape operators satisfies the equation (2.11). 


\section{Preliminaries}

Let $\left(M_{1}^{n}, g\right)$ be a $n$-dimensional Lorentz hypersurface isometrically immersed in a $n+1$-dimensional pseudoEuclidean space $\left(E_{1}^{n+1}, \bar{g}\right)$ and $g=\bar{g}_{\mid M_{1}^{n}}$. We denote by $\xi$ unit normal vector to $M_{1}^{n}$ with $\bar{g}(\xi, \xi)=1$.

Let $\bar{\nabla}$ and $\nabla$ denote linear connections on $E_{1}^{n+1}$ and $M_{1}^{n}$, respectively. Then, the Gauss and Weingarten formulae are given by

$$
\begin{gathered}
\bar{\nabla}_{X} Y=\nabla_{X} Y+h(X, Y), \quad \forall X, Y \in \Gamma\left(T M_{1}^{n}\right), \\
\bar{\nabla}_{X} \xi=-S_{\xi} X, \quad \forall \xi \in \Gamma\left(T M_{1}^{n}\right)^{\perp},
\end{gathered}
$$

where $h$ is the second fundamental form and $S$ is the shape operator. It is well known that the second fundamental form $h$ and shape operator $S$ are related by

$$
\bar{g}(h(X, Y), \xi)=g\left(S_{\xi} X, Y\right) .
$$

The mean curvature vector is given by

$$
\vec{H}=\frac{1}{n} \text { trace } h .
$$

The Gauss and Codazzi equations are given by

$$
\begin{gathered}
R(X, Y) Z=g(S Y, Z) S X-g(S X, Z) S Y, \\
\left(\nabla_{X} S\right) Y=\left(\nabla_{Y} S\right) X,
\end{gathered}
$$

respectively, where $R$ is the curvature tensor, $S=S_{\xi}$ for some unit normal vector field $\xi$ and

$$
\left(\nabla_{X} S\right) Y=\nabla_{X}(S Y)-S\left(\nabla_{X} Y\right)
$$

for all $X, Y, Z \in \Gamma\left(T M_{1}^{n}\right)$.

By comparing the tangential and normal components in biharmonic equation $\triangle \vec{H}=0$, the necessary and sufficient conditions for $M_{1}^{n}$ to have proper mean curvature in $E_{1}^{n+1}$ are

$$
\triangle H+H \operatorname{trace} S^{2}=0
$$

and

$$
S(\operatorname{grad} H)+\frac{n}{2} H \operatorname{grad} H=0,
$$

where $H$ denotes the mean curvature. Also, the Laplace operator $\triangle$ of a scalar valued function $f$ is given by [3]

$$
\triangle f=-\sum_{i=1}^{n} \epsilon_{i}\left(e_{i} e_{i} f-\nabla_{e_{i}} e_{i} f\right)
$$

where $\left\{e_{1}, e_{2}, \ldots, e_{n}\right\}$ is an orthonormal local tangent frame on $M_{1}^{n}$ with $\epsilon_{i}= \pm 1$.

A vector $X$ in $E_{s}^{n+1}$ is called spacelike, timelike or lightlike according as $\bar{g}(X, X)>0, \bar{g}(X, X)<0$ or $\bar{g}(X, X)=0$, respectively. A non-degenerate hypersurface $M_{r}^{n}$ of $E_{s}^{n+1}$ is called Riemannian or pseudoRiemannian according as the induced metric on $M_{r}^{n}$ from the indefinite metric on $E_{s}^{n+1}$ is definite or indefinite. A shape operator of pseudo-Riemannian hypersurfaces is not diagonalizable always unlike the Riemannian hypersurfaces.

It was proved in $[16,15]$ that the canonical form of the non-diagonal shape operator of $M_{1}^{n}$ in $E_{1}^{n+1}$ having minimal polynomial $(y-\lambda)^{2}\left(y-\lambda_{1}\right)\left(y-\lambda_{n}\right)$ with three distinct real eigen values takes the form

$$
S=\left(\begin{array}{ccccccccc}
\lambda & 0 & & & & & & & 0 \\
1 & \lambda & & & & & & & \\
& & \lambda & & & & & & \\
& & & \ldots & & & & & \\
& & & & \lambda_{1} & & & & \\
& & & & & \ldots & & & \\
& & & & & & \lambda_{n} & \ldots & \\
0 & & & & & & & & \lambda_{n}
\end{array}\right)
$$


with respect to some suitable pseudo-orthonormal frame of the tangent bundle.

\section{Biharmonic Lorentz hypersurfaces in $E_{1}^{n+1}$ with non-diagonal shape operator}

Let $M_{1}^{n}$ be a biharmonic Lorentz hypersurface in $E_{1}^{n+1}$ with proper mean curvature vector field having non-diagonal shape operator given by (2.11). Also, we assume that mean curvature is not constant and $\operatorname{grad} H \neq 0$. Assuming non constant mean curvature implies the existence of an open connected subset $U$ of $M_{1}^{n}$, with $\operatorname{grad}_{p} H \neq 0$, for all $p \in U$. The shape operator $S$ of a biharmonic Lorentz hypersurface given by (2.11) having the three distinct eigen values $\lambda, \lambda_{1}$ and $\lambda_{n}$ with multiplicities $r, s$ and $t$ respectively, and with minimal polynomial $(y-\lambda)^{2}\left(y-\lambda_{1}\right)\left(y-\lambda_{n}\right)$ can be written as

$$
S\left(e_{1}\right)=\lambda e_{1}+e_{2}, \quad S\left(e_{2}\right)=\lambda e_{2}, \quad S\left(e_{A}\right)=\lambda e_{A}, \quad S\left(e_{B}\right)=\lambda_{1} e_{B}, \quad S\left(e_{C}\right)=\lambda_{n} e_{C},
$$

with respect to pseudo orthonormal basis of vector fields $\left\{e_{1}, e_{2}, \ldots, e_{n}\right\}$ of $T_{p} M_{1}^{n}$, satisfying

$$
g\left(e_{1}, e_{2}\right)=-1, \quad g\left(e_{i}, e_{i}\right)=1,
$$

and

$$
g\left(e_{1}, e_{1}\right)=g\left(e_{2}, e_{2}\right)=g\left(e_{1}, e_{i}\right)=g\left(e_{2}, e_{i}\right)=g\left(e_{i}, e_{j}\right)=0,
$$

for $i \neq j$ and $i, j=3,4, \ldots, n$, and $A=3,4, \ldots, r, B=r+1, r+2, \ldots, r+s, C=r+s+1, r+s+2, \ldots, r+$ $s+t=n$.

We write

$$
\nabla_{e_{\beta}} e_{\gamma}=\sum_{k=1}^{n} \omega_{\beta \gamma}^{k} e_{k}, \quad \beta, \gamma=1,2,3, \ldots, n .
$$

Taking covariant derivatives of (3.2) and (3.3) with respect to $e_{k}$ and using (3.4), we find

$$
\omega_{k 1}^{1}=-\omega_{k 2}^{2}, \quad \omega_{k i}^{i}=\omega_{k 1}^{2}=\omega_{k 2}^{1}=0, \quad \omega_{k 1}^{i}=\omega_{k i}^{2}, \quad \omega_{k 2}^{i}=\omega_{k i}^{1}, \quad \omega_{k i}^{j}=-\omega_{k j}^{i},
$$

for $i \neq j, \quad i, j=3,4, \ldots, n$, and $k=1,2, \ldots, n$.

Now onwards, we take

$$
\begin{array}{ll}
A \neq \widetilde{A}, & A, \widetilde{A}=3,4, \ldots, r, \\
B \neq \widetilde{B}, & B, \widetilde{B}=r+1, r+2, \ldots, r+s, \\
C \neq \widetilde{C}, & C, \widetilde{C}=r+s+1, r+s+2, \ldots, r+s+t=n .
\end{array}
$$

Putting $X=e_{1}, Y=e_{2}$ in (2.6), and using (2.7) and (3.1), gives

$$
\begin{aligned}
& e_{1}(\lambda) e_{2}+\lambda \sum_{p \neq 1} \omega_{12}^{p} e_{p}-\omega_{12}^{2}\left(\lambda e_{2}\right)-\sum_{A=3}^{r} \omega_{12}^{A}\left(\lambda e_{A}\right)-\sum_{B=r+1}^{r+s} \omega_{12}^{B}\left(\lambda_{1} e_{B}\right) \\
& -\sum_{C=r+s+1}^{n} \omega_{12}^{C}\left(\lambda_{n} e_{C}\right)=e_{2}(\lambda) e_{1}+\lambda \sum_{p \neq 2} \omega_{21}^{p} e_{p}+\sum_{p \neq 1} \omega_{22}^{p} e_{p}-\omega_{21}^{1}\left(\lambda e_{1}+e_{2}\right) \\
& -\sum_{A=3}^{r} \omega_{21}^{A}\left(\lambda e_{A}\right)-\sum_{B=r+1}^{r+s} \omega_{21}^{B}\left(\lambda_{1} e_{B}\right)-\sum_{C=r+s+1}^{n} \omega_{21}^{C}\left(\lambda_{n} e_{C}\right),
\end{aligned}
$$

whereby, taking inner product with $e_{2}, e_{A}$, we obtain

$$
e_{2}(\lambda)=0,
$$

and

$$
\omega_{22}^{A}=0
$$

respectively.

Putting $X=e_{1}, Y=e_{B}$ in (2.6), and using (2.7) and (3.1), gives

$$
\begin{aligned}
& e_{1}\left(\lambda_{1}\right) e_{B}+\lambda_{1} \sum_{p \neq B} \omega_{1 B}^{p} e_{p}-\omega_{1 B}^{1}\left(\lambda e_{1}+e_{2}\right)-\omega_{1 B}^{2}\left(\lambda e_{2}\right)-\sum_{A=3}^{r} \omega_{1 B}^{A}\left(\lambda e_{A}\right)- \\
& \sum_{\widetilde{B}=r+1}^{r+s} \omega_{1 B}^{\widetilde{B}}\left(\lambda_{1} e_{\widetilde{B}}\right)-\sum_{C=r+s+1}^{n} \omega_{1 B}^{C}\left(\lambda_{n} e_{C}\right)=e_{B}(\lambda) e_{1}+\lambda \sum_{p \neq 2} \omega_{B 1}^{p} e_{p}+\sum_{p \neq 1} \omega_{B 2}^{p} e_{p} \\
& -\omega_{B 1}^{1}\left(\lambda e_{1}+e_{2}\right)-\sum_{A=3}^{r} \omega_{B 1}^{A}\left(\lambda e_{A}\right)-\sum_{m=r+1}^{r+s} \omega_{B 1}^{m}\left(\lambda_{1} e_{m}\right)-\sum_{C=r+s+1}^{n} \omega_{B 1}^{C}\left(\lambda_{n} e_{C}\right),
\end{aligned}
$$

whereby, taking inner product with $e_{2}, e_{A}, e_{\widetilde{B}}$ and $e_{C}$, we get

$$
e_{B}(\lambda)=\left(\lambda_{1}-\lambda\right) \omega_{1 B}^{1},
$$




$$
\begin{gathered}
\left(\lambda_{1}-\lambda\right) \omega_{1 B}^{A}=\omega_{B 2}^{A}, \\
\left(\lambda-\lambda_{1}\right) \omega_{B 1}^{\widetilde{B}}+\omega_{B 2}^{\widetilde{B}}=0,
\end{gathered}
$$

and

$$
\left(\lambda_{1}-\lambda_{n}\right) \omega_{1 B}^{C}=\left(\lambda-\lambda_{n}\right) \omega_{B 1}^{C}+\omega_{B 2}^{C}
$$

respectively.

Putting $X=e_{1}, Y=e_{C}$ in (2.6), and using (2.7) and (3.1), gives

$e_{1}\left(\lambda_{n}\right) e_{C}+\lambda_{1} \sum_{p \neq C} \omega_{1 C}^{p} e_{p}-\omega_{1 C}^{1}\left(\lambda e_{1}+e_{2}\right)-\omega_{1 C}^{2}\left(\lambda e_{2}\right)-\sum_{A=3}^{r} \omega_{1 C}^{A}\left(\lambda e_{A}\right)-$

$\sum_{B=r+1}^{r+s} \omega_{1 C}^{B}\left(\lambda_{1} e_{B}\right)-\sum_{\widetilde{C}=r+s+1}^{n} \omega_{1 C}^{\widetilde{C}}\left(\lambda_{n} e_{\widetilde{C}}\right)=e_{C}(\lambda) e_{1}+\lambda \sum_{p \neq 2} \omega_{C 1}^{p} e_{p}+\sum_{p \neq 1} \omega_{C 2}^{p} e_{p} \quad$ whereby, taking in$-\omega_{C 1}^{1}\left(\lambda e_{1}+e_{2}\right)-\sum_{A=3}^{r} \omega_{C 1}^{A}\left(\lambda e_{A}\right)-\sum_{B=r+1}^{r+s} \omega_{C 1}^{B}\left(\lambda_{1} e_{B}\right)-\sum_{m=r+s+1}^{n} \omega_{C 1}^{m}\left(\lambda_{n} e_{m}\right)$,

ner product with $e_{2}, e_{A}$, and $e_{C}$, we have

$$
\begin{gathered}
e_{C}(\lambda)=\left(\lambda_{n}-\lambda\right) \omega_{1 C}^{1}, \\
\left(\lambda_{n}-\lambda\right) \omega_{1 C}^{A}=\omega_{C 2}^{A},
\end{gathered}
$$

and

$$
e_{1}\left(\lambda_{n}\right)=\left(\lambda-\lambda_{n}\right) \omega_{C 1}^{C}+\omega_{C 2}^{C},
$$

respectively.

Putting $X=e_{2}, Y=e_{B}$ in (2.6) and using (2.7) and (3.1), we get

$$
\begin{aligned}
& e_{2}\left(\lambda_{1}\right) e_{B}+\lambda_{1} \sum_{p \neq B} \omega_{2 B}^{p} e_{p}-\omega_{2 B}^{1}\left(\lambda e_{1}+e_{2}\right)-\omega_{2 B}^{2}\left(\lambda e_{2}\right)-\sum_{A=3}^{r} \omega_{2 B}^{A}\left(\lambda e_{A}\right)- \\
& \sum_{\widetilde{B}=r+1}^{r+s} \omega_{2 B}^{\mathbb{B}}\left(\lambda_{1} e_{\widetilde{B}}\right)-\sum_{C=r+s+1}^{n} \omega_{2 B}^{C}\left(\lambda_{n} e_{C}\right)=e_{B}(\lambda) e_{2}+\lambda \sum_{p \neq 1} \omega_{B 2}^{p} e_{p}-\omega_{B 2}^{2}\left(\lambda e_{2}\right) \\
& -\sum_{A=3}^{r} \omega_{B 2}^{A}\left(\lambda e_{A}\right)-\sum_{m=r+1}^{r+s} \omega_{B 2}^{m}\left(\lambda_{1} e_{m}\right)-\sum_{C=r+s+1}^{n} \omega_{B 2}^{C}\left(\lambda_{n} e_{C}\right),
\end{aligned}
$$

whereby, taking inner product with $e_{1}, e_{2}, e_{A}, e_{B}, e_{\widetilde{B}}$, and $e_{C}$, we find

$$
\begin{gathered}
e_{B}(\lambda)=\left(\lambda_{1}-\lambda\right) \omega_{2 B}^{2}-\omega_{2 B}^{1}, \\
\omega_{2 B}^{1}=0, \\
\omega_{2 B}^{A}=0, \\
\left(\lambda-\lambda_{1}\right) \omega_{B 2}^{B}=e_{2}\left(\lambda_{1}\right), \\
\omega_{B 2}^{\widetilde{B}}=0,
\end{gathered}
$$

and

$$
\left(\lambda_{1}-\lambda_{n}\right) \omega_{2 B}^{C}=\left(\lambda-\lambda_{n}\right) \omega_{B 2}^{C},
$$

respectively.

Putting $X=e_{2}, Y=e_{C}$ in (2.6), and using (2.7) and (3.1), gives

$$
\begin{aligned}
& e_{2}\left(\lambda_{n}\right) e_{C}+\lambda_{n} \sum_{p \neq C} \omega_{2 C}^{p} e_{p}-\omega_{2 C}^{1}\left(\lambda e_{1}+e_{2}\right)-\omega_{2 C}^{2}\left(\lambda e_{2}\right)-\sum_{A=3}^{r} \omega_{2 C}^{A}\left(\lambda e_{A}\right)- \\
& \sum_{B=r+1}^{r+s} \omega_{2 C}^{B}\left(\lambda_{1} e_{B}\right)-\sum_{\widetilde{C}=r+s+1}^{n} \omega_{2 C}^{\widetilde{C}}\left(\lambda_{n} e_{\widetilde{C}}\right)=e_{C}(\lambda) e_{2}+\lambda \sum_{p \neq 1} \omega_{C 2}^{p} e_{p}-\omega_{C 2}^{2}\left(\lambda e_{2}\right) \\
& -\sum_{A=3}^{r} \omega_{C 2}^{A}\left(\lambda e_{A}\right)-\sum_{B=r+1}^{r+s} \omega_{C 2}^{B}\left(\lambda_{1} e_{B}\right)-\sum_{m=r+s+1}^{n} \omega_{C 2}^{m}\left(\lambda_{n} e_{m}\right),
\end{aligned}
$$

whereby, taking inner product with $e_{1}, e_{2}, e_{A}, e_{C}$ and $e_{\widetilde{C}}$, we obtain

$$
\begin{gathered}
\left(\lambda_{n}-\lambda\right) \omega_{2 C}^{2}=e_{C}(\lambda)+\omega_{2 C}^{1}, \\
\omega_{2 C}^{1}=0, \\
\omega_{2 C}^{A}=0, \\
\left(\lambda-\lambda_{n}\right) \omega_{C 2}^{C}=e_{2}\left(\lambda_{n}\right),
\end{gathered}
$$

and

$$
\omega_{C 2}^{\widetilde{C}}=0
$$


respectively.

Putting $X=e_{A}, Y=e_{B}$ in (2.6), and using (2.7) and (3.1), gives

$$
\begin{aligned}
& e_{A}\left(\lambda_{1}\right) e_{B}+\lambda_{1} \sum_{p \neq B} \omega_{A B}^{p} e_{p}-\omega_{A B}^{1}\left(\lambda e_{1}+e_{2}\right)-\omega_{A B}^{2}\left(\lambda e_{2}\right)-\sum_{m=3}^{r} \omega_{A B}^{m}\left(\lambda e_{m}\right)- \\
& \sum_{\widetilde{B}=r+1}^{r+s} \omega_{A B}^{\widetilde{B}}\left(\lambda_{1} e_{\widetilde{B}}\right)-\sum_{C=r+s+1}^{n} \omega_{A B}^{C}\left(\lambda_{n} e_{C}\right)=e_{B}(\lambda) e_{A}+\lambda \sum_{p \neq A} \omega_{B A}^{p} e_{p}-\omega_{B A}^{1} \\
& \left(\lambda e_{1}+e_{2}\right)-\omega_{B A}^{2}\left(\lambda e_{2}\right)-\sum_{\widetilde{A}=3}^{r} \omega_{B A}^{\widetilde{A}}\left(\lambda e_{\widetilde{A}}\right)-\sum_{m=r+1}^{r+s} \omega_{B A}^{m}\left(\lambda_{1} e_{m}\right) \\
& -\sum_{C=r+s+1}^{n} \omega_{B A}^{C}\left(\lambda_{n} e_{C}\right),
\end{aligned}
$$

whereby, taking inner product with $e_{2}, e_{A}, e_{\widetilde{A}}, e_{B}, e_{\widetilde{B}}$, and $e_{C}$, we get

$$
\begin{gathered}
\omega_{A B}^{1}=0, \\
\left(\lambda_{1}-\lambda\right) \omega_{A B}^{A}=e_{B}(\lambda), \\
\omega_{A B}^{\widetilde{A}}=0, \\
\left(\lambda-\lambda_{1}\right) \omega_{B A}^{B}=e_{A}\left(\lambda_{1}\right), \\
\omega_{B A}^{\widetilde{B}}=0,
\end{gathered}
$$

and

$$
\left(\lambda_{1}-\lambda_{n}\right) \omega_{A B}^{C}=\left(\lambda-\lambda_{n}\right) \omega_{B A}^{C},
$$

respectively.

Putting $X=e_{A}, Y=e_{C}$ in (2.6), and using (2.7) and (3.1), gives

$$
\begin{aligned}
& e_{A}\left(\lambda_{n}\right) e_{C}+\lambda_{n} \sum_{p \neq C} \omega_{A C}^{p} e_{p}-\omega_{A C}^{1}\left(\lambda e_{1}+e_{2}\right)-\omega_{A C}^{2}\left(\lambda e_{2}\right)-\sum_{m=3}^{r} \omega_{A C}^{m}\left(\lambda e_{m}\right)- \\
& \sum_{B=r+1}^{r+s} \omega_{A C}^{B}\left(\lambda_{1} e_{B}\right)-\sum_{\widetilde{C}=r+s+1}^{n} \omega_{A C}\left(\lambda_{n} e_{\widetilde{C}}\right)=e_{C}(\lambda) e_{A}+\lambda \sum_{p \neq A} \omega_{C A}^{p} e_{p}-\omega_{C A}^{1} \\
& \left(\lambda e_{1}+e_{2}\right)-\omega_{C A}^{2}\left(\lambda e_{2}\right)-\sum_{\widetilde{A}=3}^{r} \omega_{C A}^{\widetilde{A}}\left(\lambda e_{\widetilde{A}}\right)-\sum_{B=r+1}^{r+s} \omega_{C A}^{B}\left(\lambda_{1} e_{B}\right) \\
& -\sum_{m=r+s+1}^{n} \omega_{C A}^{m}\left(\lambda_{n} e_{m}\right),
\end{aligned}
$$

whereby, taking inner product with $e_{2}, e_{A}, e_{\widetilde{A}}, e_{B}$ and $e_{C}$, we find

$$
\begin{gathered}
\omega_{A C}^{1}=0, \\
\left(\lambda_{n}-\lambda\right) \omega_{A C}^{A}=e_{C}(\lambda), \\
\omega_{A C}^{\widetilde{A}}=0, \\
\left(\lambda_{n}-\lambda_{1}\right) \omega_{A C}^{B}=\left(\lambda-\lambda_{1}\right) \omega_{C A}^{B},
\end{gathered}
$$

and

$$
\left(\lambda-\lambda_{n}\right) \omega_{C A}^{C}=e_{A}\left(\lambda_{n}\right),
$$

respectively.

Putting $X=e_{B}, Y=e_{C}$ in (2.6), and using (2.7) and (3.1), gives

$$
\begin{aligned}
& e_{B}\left(\lambda_{n}\right) e_{C}+\lambda_{n} \sum_{p \neq C} \omega_{B C}^{p} e_{p}-\omega_{B C}^{1}\left(\lambda e_{1}+e_{2}\right)-\omega_{B C}^{2}\left(\lambda e_{2}\right)-\sum_{A=3}^{r} \omega_{B C}^{A}\left(\lambda e_{A}\right)- \\
& \sum_{m=r+1}^{r+s} \omega_{B C}^{m}\left(\lambda_{1} e_{m}\right)-\sum_{\widetilde{C}=r+s+1}^{n} \omega_{B C}\left(\lambda_{n} e_{\widetilde{C}}\right)=e_{C}\left(\lambda_{1}\right) e_{B}+\lambda_{1} \sum_{p \neq B} \omega_{C B}^{p} e_{p}-\omega_{C B}^{1} \\
& \left(\lambda e_{1}+e_{2}\right)-\omega_{C B}^{2}\left(\lambda e_{2}\right)-\sum_{A=3}^{r} \omega_{C B}^{A}\left(\lambda e_{A}\right)-\sum_{\widetilde{B}=r+1}^{r+s} \omega_{C B}^{\widetilde{B}}\left(\lambda_{1} e_{\widetilde{B}}\right) \\
& -\sum_{m=r+s+1}^{n} \omega_{C B}^{m}\left(\lambda_{n} e_{m}\right),
\end{aligned}
$$

whereby, taking inner product with $e_{1}, e_{2}, e_{B}, e_{\widetilde{B}}$ and $e_{C}$, we obtain

$$
\begin{gathered}
\left(\lambda_{n}-\lambda\right) \omega_{B C}^{2}-\omega_{B C}^{1}=\left(\lambda_{1}-\lambda\right) \omega_{C B}^{2}-\omega_{C B}^{1}, \\
\left(\lambda_{n}-\lambda\right) \omega_{B C}^{1}=\left(\lambda_{1}-\lambda\right) \omega_{C B}^{1}, \\
\left(\lambda_{n}-\lambda_{1}\right) \omega_{B C}^{B}=e_{C}\left(\lambda_{1}\right), \\
\omega_{B C}^{\widetilde{B}}=0
\end{gathered}
$$


and

$$
\left(\lambda_{1}-\lambda_{n}\right) \omega_{C B}^{C}=e_{B}\left(\lambda_{n}\right)
$$

respectively.

Similarly, evaluating $g\left(\left(\nabla_{e_{1}} S\right) e_{A}, e_{2}\right)=g\left(\left(\nabla_{e_{A}} S\right) e_{1}, e_{2}\right), \quad g\left(\left(\nabla_{e_{B}} S\right) e_{\widetilde{B}}, e_{\widetilde{B}}\right)=g\left(\left(\nabla_{e_{\widetilde{B}}} S\right) e_{B}, e_{\widetilde{B}}\right), \quad$ and $g\left(\left(\nabla_{e_{C}} S\right) e_{\widetilde{C}}, e_{\widetilde{C}}\right)=g\left(\left(\nabla_{e_{\widetilde{C}}} S\right) e_{C}, e_{\widetilde{C}}\right)$, and using (2.7) and (3.1), we get

$$
\begin{gathered}
e_{A}(\lambda)=0, \\
e_{B}\left(\lambda_{1}\right)=0,
\end{gathered}
$$

and

$$
e_{C}\left(\lambda_{n}\right)=0
$$

respectively.

Now, we consider the following cases of $\operatorname{grad} H$ viz. space like and light like depending upon preferred direction to study biharmonic Lorentz hypersurfaces in $E_{1}^{n+1}$ with non-diagonal shape operator given by (2.11). It is obvious from (2.9) that $\operatorname{grad} H$ is an eigenvector of the shape operator $S$ with the corresponding eigenvalues $-\frac{n H}{2}$.

Let grad $H$ be light like: Assuming grad $H$ in the direction of $e_{2}$, we can write grad $H=-e_{1}(H) e_{2}$. From (2.9), (2.4) and (3.1), we get

$$
\lambda=-\frac{n H}{2} \quad \text { and } \quad \lambda_{1}=\frac{n H(n-s-t+2)}{2 s}-\frac{t}{s} \lambda_{n} .
$$

Since $\operatorname{grad} H=-e_{1}(H) e_{2}$, therefore, using (3.45), we have

$$
e_{1}(H) \neq 0, \quad e_{l}(H)=0, \quad e_{l}(\lambda)=0, \quad l=2,3, \ldots, n .
$$
find

Using (3.4), (3.46) and the fact that $\left[e_{l} e_{q}\right](H)=0=\nabla_{e_{p}} e_{q}(H)-\nabla_{e_{q}} e_{l}(H)$, for $l \neq q$ and $l, q=2,3, \ldots, n$, we

$$
\omega_{l q}^{1}=\omega_{q l}^{1} .
$$

First, we consider the case of three distinct eigenvalues viz.

Case I: Let $\lambda-\lambda_{1} \neq 0, \lambda_{n}-\lambda_{1} \neq 0$ and $\lambda-\lambda_{n} \neq 0$.

Using (3.26), (3.32), (3.47) and (3.5), we have

$$
\omega_{B A}^{1}=\omega_{B 2}^{A}=\omega_{C A}^{1}=\omega_{C 2}^{A}=0 .
$$

From (3.7), (3.19), (3.25) and (3.5), we get

$$
\omega_{2 A}^{1}=\omega_{B \widetilde{B}}^{1}=\omega_{C \widetilde{C}}^{1}=0 .
$$

Also, using (3.8), (3.12), (3.15), (3.16), (3.21), (3.22), (3.46) and (3.5), we find

$$
\omega_{1 B}^{1}=\omega_{1 C}^{1}=\omega_{2 B}^{2}=\omega_{2 C}^{2}=0 .
$$

Using (3.38), (3.47) and (3.5), we obtain

$$
\omega_{C B}^{1}=\omega_{B C}^{1}=\omega_{C 2}^{B}=\omega_{B 2}^{C}=0 .
$$

Now, from (3.9), (3.13), (3.48), (3.20), (3.51) and (3.5), we have

$$
\omega_{1 B}^{A}=\omega_{1 C}^{A}=\omega_{2 B}^{C}=\omega_{2 C}^{B}=0 .
$$

Now, we have the following: 
Lemma 3.1. Let $M_{1}^{n}$ be a biharmonic Lorentz hypersurface with non-constant mean curvature in the pseudo Euclidean space $E_{1}^{n+1}$, having the non-diagonal shape operator given by (2.11). If gradH is light like and in the direction of $e_{2}$, then

$$
\begin{gathered}
\nabla_{e_{1}} e_{B}=\sum_{p \neq 1, A, B} \omega_{1 B}^{p} e_{p}, \nabla_{e_{2}} e_{A}=\sum_{p \neq 1, A} \omega_{2 A}^{p} e_{p}, \quad \nabla_{e_{2}} e_{B}=\sum_{p=r+1}^{r+s} \omega_{2 B}^{p} e_{p}, \\
\nabla_{e_{2}} e_{C}=\sum_{p=r+s+1}^{n} \omega_{2 C}^{p} e_{p}, \quad \nabla_{e_{A}} e_{B}=\sum_{p \neq 1, B} \omega_{A B}^{p} e_{p}, \quad \nabla_{e_{A}} e_{C}=\sum_{p \neq 1, C} \omega_{A C}^{p} e_{p}, \nabla_{e_{B}} e_{1}=\sum_{p \neq 2} \omega_{B 1}^{p} e_{p}, \\
\nabla_{e_{B}} e_{2}=\sum_{p \neq 1, A, \widetilde{B}, C} \omega_{B 2}^{p} e_{p}, \quad \nabla_{e_{B}} e_{B}=\sum_{p \neq B} \omega_{B B}^{p} e_{p}, \quad \nabla_{e_{B}} e_{C}=\sum_{p \neq 1, C} \omega_{B C}^{p} e_{p}, \\
\nabla_{e_{C}} e_{1}=\sum_{p \neq 2} \omega_{C 1}^{p} e_{p}, \quad \nabla_{e_{C}} e_{2}=\sum_{p \neq 1, A, B, \widetilde{C}} \omega_{C 2}^{p} e_{p}, \quad \nabla_{e_{C}} e_{B}=\sum_{p \neq 1, B} \omega_{C B}^{p} e_{p}, \\
\nabla_{e_{C}} e_{C}=\sum_{p \neq C} \omega_{C C}^{p} e_{p}, \quad \nabla_{e_{B}} e_{\widetilde{B}}=\sum_{p \neq 1, \widetilde{B}} \omega_{B \widetilde{B}}^{p} e_{p}, \quad \nabla_{e_{C}} e_{\widetilde{C}}=\sum_{p \neq 1, \widetilde{C}} \omega_{C \widetilde{C}}^{p} e_{p} .
\end{gathered}
$$

Now, computing $g\left(R\left(e_{2}, e_{B}\right) e_{B}, e_{2}\right), g\left(R\left(e_{2}, e_{C}\right) e_{C}, e_{2}\right)$, using (2.5) and Lemma 3.1, we obtain

$$
e_{2}\left(\omega_{B B}^{1}\right)+\omega_{B B}^{1}\left(\omega_{21}^{1}+\omega_{B B}^{1}\right)=0, \quad e_{2}\left(\omega_{C C}^{1}\right)+\omega_{C C}^{1}\left(\omega_{21}^{1}+\omega_{C C}^{1}\right)=0 .
$$

Adding (3.18) and (3.24), and using (3.45), (3.46) and (3.5) therein, we get

$$
\left\{\frac{n(n-t+2) H}{2}-t \lambda_{n}\right\} \omega_{B B}^{1}+t\left\{\frac{n H}{2}+\lambda_{n}\right\} \omega_{C C}^{1}=0 .
$$

Acting on (3.54) with $e_{2}$ and using (3.53), we find

$$
2 t e_{2}\left(\lambda_{n}\right)\left[\omega_{C C}^{1}-\omega_{B B}^{1}\right]=0,
$$

which implies either $e_{2}\left(\lambda_{n}\right)=0$ or $\omega_{C C}^{1}=\omega_{B B}^{1}$. In both cases, using (3.18), (3.24), (3.54) and (3.5), we have

$$
\omega_{B B}^{1}=\omega_{C C}^{1}=\omega_{B 2}^{B}=\omega_{C 2}^{C}=0 .
$$

Now, computing $g\left(R\left(e_{B}, e_{1}\right) e_{B}, e_{2}\right), g\left(R\left(e_{C}, e_{1}\right) e_{C}, e_{2}\right)$ and using (2.5), Lemma 3.1 and (3.45), we obtain

$$
\sum_{A=3}^{r} \omega_{B B}^{A} \omega_{1 A}^{1}=-\frac{n H}{2}\left\{\frac{n H(n-s-t+2)}{2 s}-\frac{t}{s} \lambda_{n}\right\}
$$

and

$$
\sum_{A=3}^{r} \omega_{C C}^{A} \omega_{1 A}^{1}=-\frac{n H}{2} \lambda_{n}
$$

Now, adding (3.29) and (3.36), and using (3.45), (3.46) and (3.5) therein, we get

$$
\left\{\frac{n(n-t+2) H}{2}-t \lambda_{n}\right\} \omega_{B B}^{A}+t\left\{\frac{n H}{2}+\lambda_{n}\right\} \omega_{C C}^{A}=0 .
$$

Since $A$ varies from 3 to $r$, therefore (3.56), (3.57) and (3.58) is valid for $r>2$. Multiplying (3.58) by $\omega_{1 A}^{1}$ and taking summation over $A$ and then using (3.56) and (3.57), we get

$$
4(s+t) t \lambda_{n}^{2}-4 n(n-s-t+2) t H \lambda_{n}+n^{2}(n-t+2)(n-s-t+2) H^{2}=0 .
$$

Now, from (3.59), we find $\lambda_{n}$ imaginary as discriminant $D=-16 n^{2} H^{2}(n-s-t+2)\left(n s t+2 t s+2 t^{2}\right)<0$. Therefore, a contradiction, hence, $r>2$ is not possible.

Now, for $r=2,(3.56)$ and (3.57) reduce to

$$
-\frac{n H}{2}\left\{\frac{n H(n-s-t+2)}{2 s}-\frac{t}{s} \lambda_{n}\right\}=0,
$$


and

$$
-\frac{n H}{2} \lambda_{n}=0 .
$$

Hence, From (3.60) and (3.61), we obtain that $H=0$.

Case II: Let either $\lambda-\lambda_{1}=0$ or $\lambda_{n}-\lambda_{1}=0$ or $\lambda-\lambda_{n}=0$. Then, from (3.45), we find that each eigen value $\lambda, \lambda_{1}$ and $\lambda_{n}$ are proportional to $H$. So, from (3.46), we have

$$
e_{l}(\lambda)=e_{l}\left(\lambda_{1}\right)=e_{l}\left(\lambda_{n}\right)=0, \quad \text { for } \quad l=2,3, \ldots, n .
$$

If $\lambda=\lambda_{1}$, then using (3.36), (3.62) and (3.5), we get

$$
\omega_{C A}^{C}=\omega_{C C}^{A}=0 .
$$

Using (3.63) and computing $g\left(R\left(e_{C}, e_{1}\right) e_{C}, e_{2}\right)$, we get that $H=0$.

Now, if $\lambda_{1}=\lambda_{n}$ or $\lambda=\lambda_{n}$, in both cases from (3.29), (3.62) and (3.5), we obtain $\omega_{B A}^{B}=\omega_{B B}^{A}=0$. Evaluating $g\left(R\left(e_{B}, e_{1}\right) e_{B}, e_{2}\right)$, we find that $H=0$.

Combining Case I and Case II, we have:

Proposition 3.1. Let $M_{1}^{n}$ be a biharmonic Lorentz hypersurface in the pseudo Euclidean space $E_{1}^{n+1}$ having the nondiagonal shape operator given by (2.11). If gradH is light like, then $M_{1}^{n}$ is minimal.

Now, we discuss the space like case of $\operatorname{grad} H$.

Let grad $H$ be space like: In this case grad $H$ can be in the direction of $e_{A}$ or $e_{B}$ or $e_{C}$. In view of (3.42), (3.43) and (3.44), one of the multiplicities of eigen values must be one, otherwise, we get contradiction. Since $r \geq 2$, therefore either $s$ or $t$ must be one. Without loss of generality, we assume that $r \geq 2, s \geq 1, t=1$ and $\operatorname{grad} H$ is in the direction of $e_{n}$. We can write $\operatorname{grad} H=e_{n}(H) e_{n}$. Now, we have $A=3,4, \ldots, r, B=r+1, r+2, \ldots, r+s=$ $n-1$ and $C=n$. From (2.9) and (2.4), we get

$$
\lambda_{n}=-\frac{n H}{2}, \quad \text { and } \quad \lambda_{1}=\frac{3 n H}{2(n-r-1)}-\frac{r \lambda}{n-r-1} .
$$

Since $\operatorname{grad} H=e_{n}(H) e_{n}$, therefore, from (3.64), we have

$$
e_{n}(H) \neq 0, \quad e_{a}(H)=0 \quad e_{a}\left(\lambda_{n}\right)=0, \quad a=1,2, \ldots, n-1 .
$$

Using (3.4), (3.65) and the fact that $\left[e_{a} e_{b}\right](H)=0=\nabla_{e_{a}} e_{b}(H)-\nabla_{e_{b}} e_{a}(H)$, for $a \neq b$ and $a, b=1,2, \ldots, n-1$, we find

$$
\omega_{a b}^{n}=\omega_{b a}^{n} .
$$

Now, we consider the case of three distinct eigenvalues viz.

Case III: Let $\lambda-\lambda_{1} \neq 0, \lambda_{n}-\lambda_{1} \neq 0$ and $\lambda-\lambda_{n} \neq 0$.

From (3.6), (3.42), (3.64) and (3.65), we have

$$
e_{2}\left(\lambda_{1}\right)=0, \quad e_{A}\left(\lambda_{1}\right)=0 .
$$

From (3.18), (3.24), (3.29), (3.36), (3.65), (3.67) and (3.5), we get

$$
\omega_{B 2}^{B}=\omega_{B B}^{1}=\omega_{n 2}^{n}=\omega_{n n}^{1}=\omega_{B A}^{B}=\omega_{B B}^{A}=\omega_{n A}^{n}=\omega_{n n}^{A}=0 .
$$

Using (3.7), (3.16), (3.17), (3.22), (3.26) and (3.5), we have

$$
\omega_{2 A}^{1}=\omega_{22}^{B}=\omega_{2 A}^{B}=\omega_{22}^{n}=\omega_{A 2}^{B}=0 .
$$

Using (3.20), (3.32), (3.66) and (3.5), we have

$$
\omega_{2 B}^{n}=\omega_{B 2}^{n}=\omega_{2 n}^{B}=\omega_{B n}^{1}=\omega_{A 2}^{n}=\omega_{2 A}^{n}=\omega_{2 n}^{A}=0 .
$$


Also, using (3.11), (3.31), (3.70), and (3.5), we obtain

$$
\omega_{1 B}^{n}=\omega_{B 1}^{n}=\omega_{1 n}^{B}=\omega_{B n}^{2}=\omega_{A B}^{n}=\omega_{B A}^{n}=\omega_{A n}^{B}=\omega_{B n}^{A}=0 .
$$

From (3.28), (3.30), (3.34), (3.40), and (3.5), we get

$$
\omega_{A \widetilde{A}}^{B}=\omega_{B \widetilde{B}}^{A}=\omega_{A \widetilde{A}}^{n}=\omega_{B \widetilde{B}}^{n}=0 .
$$

Using (3.14), (3.41), (3.65), (3.68) and (3.5), we have

$$
\omega_{n 1}^{n}=\omega_{n n}^{2}=\omega_{n B}^{n}=\omega_{n n}^{B}=0 .
$$

From (3.35), (3.38), (3.71), (3.70), and (3.5), we get

$$
\omega_{n A}^{B}=\omega_{n B}^{1}=\omega_{n B}^{A}=\omega_{n 2}^{B}=0 .
$$

From (3.10), (3.19), (3.37), (3.70), (3.71), (3.74), and (3.5), we get

$$
\omega_{B 1}^{\widetilde{B}}=\omega_{B \widetilde{B}}^{1}=\omega_{n B}^{2}=\omega_{n 1}^{B}=0 .
$$

Now, we have the following:

Lemma 3.2. Let $M_{1}^{n}$ be a biharmonic Lorentz hypersurface in the pseudo Euclidean space $E_{1}^{n+1}$, having the non-diagonal shape operator given by (2.11). If grad $H$ is space like and in the direction of $e_{n}$, then

$$
\begin{aligned}
& \nabla_{e_{1}} e_{2}= \sum_{p \neq 1} \omega_{12}^{p} e_{p}, \quad \nabla_{e_{1}} e_{B}=\sum_{p \neq B, n} \omega_{1 B}^{p} e_{p}, \quad \nabla_{e_{1}} e_{n}=\sum_{p \neq B, n} \omega_{1 n}^{p} e_{p}, \quad \nabla_{e_{2}} e_{A}=\sum_{p \neq 1, A, B, n} \omega_{2 A}^{p} e_{p}, \\
& \nabla_{e_{2}} e_{B}= \sum_{p \neq 1, A, B, n} \omega_{2 B}^{p} e_{p}, \quad \nabla_{e_{2}} e_{n}=\omega_{2 n}^{2} e_{2}, \quad \nabla_{e_{A}} e_{B}=\sum_{p \neq 1, \widetilde{A}, B, n} \omega_{A B}^{p} e_{p}, \quad \nabla_{e_{A}} e_{\widetilde{A}}=\sum_{p \neq \widetilde{A}, B, n} \omega_{A \widetilde{A}}^{p} e_{p}, \\
& \nabla_{e_{A}} e_{n}=\sum_{p \neq 1, \widetilde{A}, B, n} \omega_{A n}^{p} e_{p}, \quad \nabla_{e_{B}} e_{1}=\sum_{p \neq 2, \widetilde{B}, n} \omega_{B 1}^{p} e_{p}, \nabla_{e_{B}} e_{2}=\sum_{p \neq 1, B, \widetilde{B}, n} \omega_{B 2}^{p} e_{p}, \\
& \nabla_{e_{B}} e_{A}= \sum_{p \neq A, B, \widetilde{B}, n} \omega_{B A}^{p} e_{p}, \quad \nabla_{e_{B}} e_{\widetilde{B}}=\sum_{p \neq 1, A, \widetilde{B}, n} \omega_{B \widetilde{B}}^{p} e_{p}, \nabla_{e_{B}} e_{B}=\sum_{p \neq 1, A, B} \omega_{B B}^{p} e_{p}, \nabla_{e_{B}} e_{n}=\omega_{B n}^{B} e_{B}, \\
& \nabla_{e_{n}} e_{1}= \sum_{p \neq 2, B, n} \omega_{n 1}^{p} e_{p}, \quad \nabla_{e_{n}} e_{2}=\sum_{p \neq 1, B, n} \omega_{n 2}^{p} e_{p}, \quad \nabla_{e_{n}} e_{A}=\sum_{p \neq A, B, n} \omega_{n A}^{p} e_{p}, \quad \nabla_{e_{n}} e_{B}=\sum_{p=r+1}^{n-1} \omega_{n B}^{p} e_{p}, \\
& \nabla_{e_{n}} e_{n}=0, \quad \nabla_{e_{1}} e_{A}=\sum_{p \neq A} \omega_{1 A}^{p} e_{p}, \quad \nabla_{e_{2}} e_{2}=\sum_{p \neq 1, B, n} \omega_{22}^{p} e_{p}, \quad \nabla_{e_{A}} e_{1}=\sum_{p \neq 2} \omega_{A 1}^{p} e_{p}, \\
& \nabla_{e_{A}} e_{2}=\sum_{p \neq 1, B, n} \omega_{A 2}^{p} e_{p}, \quad \nabla_{e_{2}} e_{1}=\sum_{p \neq 2} \omega_{21}^{p} e_{p} .
\end{aligned}
$$

Now, to find the Laplace operator, we need to construct an orthonormal basis $\left\{X_{1}, X_{2}, \ldots, X_{n}\right\}$ from the pseudo-orthonormal basis $\left\{e_{1}, e_{2}, \ldots, e_{n}\right\}$. Therefore, we take

$$
X_{1}=\frac{e_{1}+e_{2}}{\sqrt{2}}, \quad X_{2}=\frac{e_{1}-e_{2}}{\sqrt{2}}, \quad X_{i}=e_{i}, \quad i=3,4, \ldots, n .
$$

Also, using (3.64), we obtain

$$
\text { trace } S^{2}=\frac{(n-1) r}{n-r-1} \lambda^{2}+\frac{n^{2}(n-r+8)}{4(n-r-1)} H^{2}-\frac{3 n r}{n-r-1} H \lambda .
$$


Using (2.10) and (3.76) the Laplace operator for the pseudo-orthonormal basis $\left\{e_{1}, e_{2}, \ldots, e_{n}\right\}$, is given by

$$
\triangle=e_{1} e_{2}+e_{2} e_{1}-\sum_{i=3}^{n} e_{i} e_{i}-\nabla_{e_{1}} e_{2}-\nabla_{e_{2}} e_{1}+\sum_{i=3}^{n} \nabla_{e_{i}} e_{i}(H)
$$

Using (3.77), (3.78) and Lemma 3.3 in (2.8), we find

$$
\begin{array}{r}
{\left[-2 \omega_{12}^{n}+\sum_{A=3}^{r} \omega_{A A}^{n}+\sum_{B=r+1}^{n-1} \omega_{B B}^{n}\right] e_{n}(H)-e_{n} e_{n}(H)+H\left[\frac{(n-1) r}{n-r-1} \lambda^{2}+\frac{n^{2}(n-r+8)}{4(n-r-1)} H^{2}\right.} \\
\left.-\frac{3 n r}{n-r-1} H \lambda\right]=0 .
\end{array}
$$

Now, from (3.12), (3.33), (3.39), (3.8), (3.27) and (3.5), we find

$$
\omega_{A A}^{n}=-\omega_{12}^{n}, \quad \omega_{A A}^{B}=-\omega_{12}^{B}, \quad \omega_{A A}^{n}=\omega_{\widetilde{A} \widetilde{A}}^{n}, \quad \omega_{B B}^{n}=\omega_{\widetilde{B} \widetilde{B}}^{n} .
$$

Therefore, using (3.80) in (3.79), we obtain

$$
\begin{aligned}
{\left[-r \omega_{12}^{n}+(n-r-1) \omega_{B B}^{n}\right] e_{n}(H)-e_{n} e_{n}(H)+H\left[\frac{(n-1) r}{n-r-1} \lambda^{2}+\frac{n^{2}(n-r+8)}{4(n-r-1)} H^{2}\right.} & \\
\left.-\frac{3 n r}{n-r-1} H \lambda\right] & =0 .
\end{aligned}
$$

Now, we have:

Lemma 3.3. Let $M_{1}^{n}$ be a biharmonic Lorentz hypersurface in the pseudo Euclidean space $E_{1}^{n+1}$, having the non-diagonal shape operator given by (2.11). If grad $H$ is space like and in the direction of $e_{n}$. Then, $e_{B}(\lambda)=0$ for $s \geq 1$.

Proof. From (3.43) and (3.64), we get $e_{B}(\lambda)=0$ for $s>1$. Now, for $s=1$, we have $B=n-1$ and $r=n-2$. Now, putting $r=n-2$ and $B=n-1$ in (3.81), we get

$$
\left[(-n+2) \omega_{12}^{n}+\omega_{(n-1)(n-1)}^{n}\right] e_{n}(H)-e_{n} e_{n}(H)+H\left[(n-1)(n-2) \lambda^{2}+\frac{5 n^{2}}{2} H^{2}-3 n(n-2) H \lambda\right]=0 .
$$

Using (2.5), (3.5), (3.80) and Lemma 3.3, computing $g\left(R\left(e_{n-1}, e_{1}\right) e_{2}, e_{n}\right)$ and $g\left(R\left(e_{A}, e_{n-1}\right) e_{n}, e_{A}\right)$, we find

$$
e_{n-1}\left(\omega_{12}^{n}\right)+\omega_{12}^{n-1}\left(\omega_{(n-1)(n-1)}^{n}+\omega_{12}^{n}\right)-\sum_{A=3}^{r} \omega_{(n-1) A}^{1} \omega_{1 A}^{n}=0,
$$

and

$$
e_{n-1}\left(\omega_{12}^{n}\right)+\omega_{12}^{n-1}\left(\omega_{(n-1)(n-1)}^{n}+\omega_{12}^{n}\right)+2 \omega_{(n-1) A}^{1} \omega_{1 A}^{n}=0
$$

respectively.

Taking summation over $A$ from 3 to $r$ in (3.84), we find

$$
(r-2) e_{n-1}\left(\omega_{12}^{n}\right)+(r-2) \omega_{12}^{n-1}\left(\omega_{(n-1)(n-1)}^{n}+\omega_{12}^{n}\right)+2 \sum_{A=3}^{r} \omega_{(n-1) A}^{1} \omega_{1 A}^{n}=0 .
$$

Combining (3.83) and (3.85), we obtain

$$
r e_{n-1}\left(\omega_{12}^{n}\right)+r \omega_{12}^{n-1}\left(\omega_{(n-1)(n-1)}^{n}+\omega_{12}^{n}\right)=0,
$$

or,

$$
e_{n-1}\left(\omega_{12}^{n}\right)=-\omega_{12}^{n-1}\left(\omega_{(n-1)(n-1)}^{n}+\omega_{12}^{n}\right),
$$

Using (3.8), (3.64), (3.87) and (3.5) for $r=n-2$, we find

$$
e_{n-1}\left(\omega_{12}^{n}\right)=-\frac{e_{n-1}(\lambda)}{\frac{3 n H}{2}-(n-1) \lambda}\left(\omega_{(n-1)(n-1)}^{n}+\omega_{12}^{n}\right) .
$$


Using (3.64), (3.65), (3.66) and $r=n-2$ in (3.12) and (3.39), we have

$$
e_{n}(\lambda)=-\left(\frac{n H}{2}+\lambda\right) \omega_{12}^{n}
$$

and

$$
e_{n}\left(\frac{3 n H}{2}-(n-2) \lambda\right)=(2 n H-(n-2) \lambda) \omega_{(n-1)(n-1)}^{n},
$$

respectively.

Adding (3.89) and (3.90), we get

$$
\frac{3 n}{2} e_{n}(H)=-(n-2)\left(\frac{n H}{2}+\lambda\right) \omega_{12}^{n}+(2 n H-(n-2) \lambda) \omega_{(n-1)(n-1)}^{n} .
$$

Using (3.65) and Lemma 3.3, and the fact that $\left[e_{a} e_{n}\right](H)=0=\nabla_{e_{a}} e_{n}(H)-\nabla_{e_{n}} e_{a}(H)$, for $a=1,2, \ldots, n-1$, we obtain

$$
e_{a} e_{n}(H)=0 \text {. }
$$

Differentiating (3.91) with respect to $e_{n-1}$ and using (3.88), (3.89) and (3.92), we find

$$
e_{n-1}\left(\omega_{(n-1)(n-1)}^{n}\right)=\frac{2 n(n-2)(H-\lambda)\left(\omega_{(n-1)(n-1)}^{n}+\omega_{12}^{n}\right) e_{n-1}(\lambda)}{(2 n H-(n-2) \lambda)(3 n H-2(n-1) \lambda)} .
$$

Taking derivative of (3.82) along $e_{n-1}$ and using (3.88), (3.92) and (3.93), we get

$$
(n-2) e_{n-1}(\lambda)\left[2 e_{n}(H)\left(\omega_{(n-1)(n-1)}^{n}+\omega_{12}^{n}\right)+H(2(n-1) \lambda-3 n H)(2 n H-(n-2) \lambda)\right]=0 .
$$

If $e_{n-1}(\lambda) \neq 0$ in the above, then

$$
2 e_{n}(H)\left(\omega_{(n-1)(n-1)}^{n}+\omega_{12}^{n}\right)+H(2(n-1) \lambda-3 n H)(2 n H-(n-2) \lambda)=0 .
$$

Differentiating (3.94) along $e_{n-1}$ and using (3.88) and (3.93), we obtain

$$
\begin{aligned}
4(n(n-4) H-(n-2)(n-1) \lambda)\left(\omega_{(n-1)(n-1)}^{n}+\omega_{12}^{n}\right) e_{n}(H)+H[n(7 n-10) H \\
-4(n-1)(n-2) \lambda)(2 n H-(n-2) \lambda)(3 n H-2(n-1) \lambda]=0 .
\end{aligned}
$$

Eliminating $e_{n}(H)$ from (3.94) and (3.95), we get

$$
\lambda=\frac{3 n H}{2(n-1)} \Rightarrow \lambda_{1}=\frac{3 n H}{2(n-1)}=\lambda,
$$

which is a contradiction of distinct principal curvatures, consequently $e_{n-1}(\lambda)=0$. Whereby proof of Lemma is complete.

Next, we have:

Lemma 3.4. Let $M_{1}^{n}$ be a biharmonic Lorentz hypersurface in the pseudo Euclidean space $E_{1}^{n+1}$, having the non-diagonal shape operator given by (2.11). If gradH is space like and in the direction of $e_{n}$. Then, we find

$$
\begin{gathered}
e_{n}\left(\omega_{12}^{n}\right)+\left(\omega_{12}^{n}\right)^{2}=\frac{n H}{2} \lambda, \\
\omega_{B B}^{n} \omega_{12}^{n}=\lambda\left(\frac{3 n H}{2(n-r-1)}-\frac{r \lambda}{n-r-1}\right),
\end{gathered}
$$

and

$$
e_{n}\left(\omega_{B B}^{n}\right)-\left(\omega_{B B}^{n}\right)^{2}=-\frac{n H}{2}\left(\frac{3 n H}{2(n-r-1)}-\frac{r \lambda}{n-r-1}\right) .
$$

Proof. Using (3.8), (3.15), (3.16), (3.27), (3.5) and Lemma 3.4, we obtain

$$
\omega_{12}^{B}=\omega_{21}^{B}=\omega_{1 B}^{1}=\omega_{2 B}^{2}=\omega_{A B}^{A}=\omega_{A A}^{B}=0 .
$$


Also, evaluating $g\left(\left(\nabla_{e_{1}} S\right) e_{A}, e_{\widetilde{A}}\right)=g\left(\left(\nabla_{e_{A}} S\right) e_{1}, e_{\widetilde{A}}\right)$ and $g\left(\left(\nabla_{e_{1}} S\right) e_{A}, e_{B}\right)=g\left(\left(\nabla_{e_{A}} S\right) e_{1}, e_{B}\right)$, using (2.6), (3.1) and (3.69), we get

$$
\omega_{A 2}^{\widetilde{A}}=\omega_{A \widetilde{A}}^{1}=0 \quad \text { and } \quad \omega_{1 A}^{B}=\omega_{A 1}^{B},
$$

respectively.

Computing $g\left(R\left(e_{n}, e_{1}\right) e_{n}, e_{2}\right)$ and $g\left(R\left(e_{n}, e_{A}\right) e_{n}, e_{A}\right)$, using (2.5), (3.68), (3.99), (3.80), (3.5) and Lemma 3.3, we find

$$
e_{n}\left(\omega_{12}^{n}\right)+\left(\omega_{12}^{n}\right)^{2}-\sum_{A=3}^{r} \omega_{n 2}^{A} \omega_{1 A}^{n}=\frac{n H}{2} \lambda,
$$

and

$$
e_{n}\left(\omega_{12}^{n}\right)+\left(\omega_{12}^{n}\right)^{2}+2 \omega_{n 2}^{A} \omega_{1 A}^{n}=\frac{n H}{2} \lambda
$$

respectively.

Now, taking summation over $A$ from 3 to $r$ in (3.102), we get

$$
(r-2) e_{n}\left(\omega_{12}^{n}\right)+(r-2)\left(\omega_{12}^{n}\right)^{2}+2 \sum_{A=3}^{r} \omega_{n 2}^{A} \omega_{1 A}^{n}=(r-2) \frac{n H}{2} \lambda .
$$

Now, combining (3.101) and (3.103), we obtain (3.96).

Next, evaluating $g\left(R\left(e_{1}, e_{B}\right) e_{B}, e_{2}\right), g\left(R\left(e_{A}, e_{B}\right) e_{B}, e_{A}\right)$ and $g\left(R\left(e_{A}, e_{1}\right) e_{2}, e_{B}\right)$, using (2.5), (3.68), (3.99), (3.100), (3.80), (3.5) and Lemma 3.3, we find

$$
\begin{gathered}
\omega_{B B}^{n} \omega_{12}^{n}-\sum_{A=3}^{r} \omega_{1 B}^{A} \omega_{B A}^{1}=\lambda\left(\frac{3 n H}{2(n-r-1)}-\frac{r \lambda}{n-r-1}\right), \\
\omega_{B B}^{n} \omega_{12}^{n}+\omega_{B B}^{2} \omega_{A A}^{1}+2 \omega_{B A}^{1} \omega_{1 B}^{A}=\lambda\left(\frac{3 n H}{2(n-r-1)}-\frac{r \lambda}{n-r-1}\right),
\end{gathered}
$$

and

$$
\omega_{A A}^{1} \omega_{1 B}^{A}=0,
$$

respectively.

From (3.106), we have either $\omega_{A A}^{1}=0$ or $\omega_{1 B}^{A}=0$. In both the cases, from (3.104) and (3.105), we get (3.97).

Similarly, evaluating $g\left(R\left(e_{n}, e_{B}\right) e_{n}, e_{B}\right)$, we obtain (3.98).

Now, we have:

Proposition 3.2. Let $M_{1}^{n}$ be a biharmonic Lorentz hypersurface in the pseudo Euclidean space $E_{1}^{n+1}$ with three distinct eigen values and having the non-diagonal shape operator given by (2.11). If gradH is space like, then $M_{1}^{n}$ is not proper biharmonic.

Proof. Using (3.64) and (3.5) in (3.12), we get

$$
e_{n}(\lambda)=-\left(\frac{n H}{2}+\lambda\right) \omega_{12}^{n}
$$

Using (3.5), (3.64) and (3.107) in (3.39), we find

$$
3 n e_{n}(H)=[n H(n-r+2)-2 r \lambda] \omega_{B B}^{n}-2 r\left(\frac{n H}{2}+\lambda\right) \omega_{12}^{n} .
$$

Now, multiplying (3.108) by $\omega_{12}^{n}$ and using (3.97), we have

$$
\left(\omega_{12}^{n}\right)^{2}\left(\frac{n H}{2}+\lambda\right)=-3 n \omega_{12}^{n} e_{n}(H)+\frac{\lambda}{n-r-1}[n H(n-r+2)-2 r \lambda]\left(\frac{3 n H}{2}-r \lambda\right) .
$$

Similarly, multiplying (3.108) by $\omega_{B B}^{n}$ and using (3.97), we obtain

$$
\left(\omega_{B B}^{n}\right)^{2}(n H(n-r+2)-2 r \lambda)=3 n \omega_{B B}^{n} e_{n}(H)+\frac{2 r \lambda}{n-r-1}\left(\frac{n H}{2}+\lambda\right)\left(\frac{3 n H}{2}-r \lambda\right) .
$$


Differentiating (3.108) along $e_{n}$, and using (3.96), (3.97), (3.98) and (3.107), we find

$$
\begin{aligned}
3 n e_{n} e_{n}(H)=e_{n}(H)\left[n(n-r+5) \omega_{B B}^{n}-n(r+6) \omega_{12}^{n}\right]-r n H \lambda\left(\frac{n H}{2}+\lambda\right) & \\
& +\frac{3 n H-2 r \lambda}{4(n-r-1)}\left[-n^{2}(n-r+2) H^{2}+2 n(r+2 n+4) H \lambda\right] .
\end{aligned}
$$

Eliminating $e_{n} e_{n}(H)$ from (3.81) and (3.111), we get

$$
e_{n}(H)\left[(n-r-4) \omega_{B B}^{n}+(3-r) \omega_{12}^{n}\right]+\frac{3 n H}{4(n-r-1)}\left[n(n-r+5) H^{2}-(2 n+8 r+4) H \lambda+4 r \lambda^{2}\right]=0 .
$$

Acting with $e_{n}$ on (3.112) and putting the value of $e_{n} e_{n}(H)$ from (3.81) and using (3.96), (3.97), (3.98) and (3.107), we find

$$
\begin{aligned}
& {\left[(n-r-4) \omega_{B B}^{n}+(3-r) \omega_{12}^{n}\right]\left[H\left\{\frac{(n-1) r}{n-r-1} \lambda^{2}-\frac{3 n r}{n-r-1} H \lambda+\frac{n^{2}(n-r+8)}{4(n-r-1)}\right\}\right]} \\
& +\left[(n-r)(n-r-4)\left(\omega_{B B}^{n}\right)^{2}-(3-r)(r+1)\left(\omega_{12}^{n}\right)^{2}+\frac{[(n-r-1)(3-r)-r(n-r-4)]}{2(n-r-1)}\right. \\
& \left.\quad\left(3 n H \lambda-2 r \lambda^{2}\right)\right] e_{n}(H)+\frac{n e_{n}(H)}{4(n-r-1)}\left[3 n(2 n-2 r+19) H^{2}+(2 r(n-r-4)-\right. \\
& \left.2(6 n+25 r+9)) H \lambda+12 r \lambda^{2}\right]+\frac{3 n}{4(n-r-1)}\left[(n+4 r+2) H^{2}-4 r H \lambda\right](n H+2 \lambda) \omega_{12}^{n}=0 .
\end{aligned}
$$

Now, multiplying (3.112) by $\omega_{12}^{n}$ and using (3.97), we have

$$
\begin{aligned}
(3-r) e_{n}(H)\left(\omega_{12}^{n}\right)^{2}=-\frac{n-r-4}{2(n-r-1)}( & \left.3 n H \lambda-2 r \lambda^{2}\right) e_{n}(H) \\
& \quad-\frac{3 n H}{4(n-r-1)}\left[n(n-r+5) H^{2}-(2 n+8 r+4) H \lambda+4 r \lambda^{2}\right] \omega_{12}^{n} .
\end{aligned}
$$

Similarly, multiplying (3.112) by $\omega_{B B}^{n}$ and using (3.97), we obtain

$$
\begin{aligned}
(n-r-4) e_{n}(H)\left(\omega_{B B}^{n}\right)^{2}=-\frac{3-r}{2(n-r-1)} & \left(3 n H \lambda-2 r \lambda^{2}\right) e_{n}(H) \\
& \quad-\frac{3 n H}{4(n-r-1)}\left[n(n-r+5) H^{2}-(2 n+8 r+4) H \lambda+4 r \lambda^{2}\right] \omega_{B B}^{n} .
\end{aligned}
$$

Using (3.114) and (3.115) in (3.113), we get

$$
\omega_{12}^{n} E+\omega_{B B}^{n} F+e_{n}(H) G=0,
$$

where

$$
\begin{aligned}
E & =H\left[\left(9 n+13 r+2 n r-2 r^{2}+45\right) n^{2} H^{2}+4 r(2 n r+r-3) \lambda^{2}-6 n r(3 n+2 r+8) H \lambda\right], \\
F & =H\left[-((n-r)(2 n-2 r+11)+32) n^{2} H^{2}-4 r((n-r)(2 n+1)-4(n-1)) \lambda^{2}\right. \\
& +6 n((n-r)(n+2 r+8)+8 r) H \lambda], \\
G & =4 r(2 n+7) \lambda^{2}+3 n^{2}(2 n-2 r+19) H^{2}+2 n((n-r)(r-6)+(3 n-35 r-30)) H \lambda .
\end{aligned}
$$

Eliminating $e_{n}(H)$ from (3.116) and (3.108), we obtain

$$
\omega_{12}^{n} f_{1}(H, \lambda)+\omega_{B B}^{n} f_{2}(H, \lambda)=0,
$$

where $f_{1}(H, \lambda)=E-\frac{r(n H+2 \lambda)}{3 n} G$ and $f_{2}(H, \lambda)=F+\frac{(n(n-r+2) H-2 r \lambda)}{3 n} G$ are the homogeneous functions of degree 3 in terms of $H$ and $\lambda$.

Multiplying (3.117) by $\omega_{12}^{n}$ and $\omega_{B B}^{n}$ and using (3.97), we obtain

$$
\left(\omega_{12}^{n}\right)^{2} f_{1}(H, \lambda)=-\frac{\lambda}{2(n-r-1)}(3 n H-2 r \lambda) f_{2}(H, \lambda),
$$


and

$$
\left(\omega_{B B}^{n}\right)^{2} f_{2}(H, \lambda)=-\frac{\lambda}{2(n-r-1)}(3 n H-2 r \lambda) f_{1}(H, \lambda)
$$

respectively.

Again, eliminating $e_{n}(H)$ from (3.112) and (3.108), we get

$$
P_{1}\left(\omega_{B B}^{n}\right)^{2}-P_{2}\left(\omega_{12}^{n}\right)^{2}+P_{3}=0,
$$

where

$$
\begin{aligned}
P_{1} & =4(n-r-1)(n-r-4)(n(n-r+2) H-2 r \lambda), \\
P_{2} & =4 r(n-r-1)(3-r)(n H+2 \lambda), \\
P_{3} & =8 r^{2}(n-2 r-1) \lambda^{3}+9 n^{3}(n-r+5) H^{3}-6 n^{2} r(2 n-2 r+13) H^{2} \lambda \\
& +4 n r\{3(n+3 r-1)+2 r(n-r-1)\} H \lambda^{2} .
\end{aligned}
$$

Now, eliminating $\omega_{12}^{n}$ and $\omega_{B B}^{n}$ from (3.120) by using (3.118) and (3.119), we obtain

$$
\lambda(3 n H-2 r \lambda)\left[\left(f_{2}(H, \lambda)\right)^{2} P_{2}-\left(f_{1}(H, \lambda)\right)^{2} P_{1}\right]+2(n-r-1) f_{1}(H, \lambda) f_{2}(H, \lambda) P_{3}=0,
$$

which is a homogeneous equation of degree 9 in terms of $H$ and $\lambda$. Here, we point out that $\lambda \neq 0$. In fact, if $\lambda=0$ then (3.121) gives $H=0$, which is contradiction to our assumption. We put $Y=\frac{H}{\lambda}$, then (3.121) will reduce to an algebraic equation in $Y$

$$
(3 n Y-2 r)\left[P_{4}-P_{5}\right]+2(n-r-1) P_{6}=0,
$$

where

$$
\begin{aligned}
P_{4} & =4 r(n-r-1)(3-r)(n Y+2)\left(g_{2}(Y)\right)^{2}, \\
P_{5} & =4(n-r-1)(n-r-4)(n(n-r+2) Y-2 r)\left(g_{1}(Y)\right)^{2}, \\
P_{6} & =\left[8 r^{2}(n-2 r-1)+9 n^{3}(n-r+5) Y^{3}-6 n^{2} r(2 n-2 r+13) Y^{2}\right. \\
& +4 n r\{3(n+3 r-1)+2 r(n-r-1)\} Y] g_{1}(Y) g_{2}(Y), \\
g_{1}(Y) & =Y\left[\left(9 n+13 r+2 n r-2 r^{2}+45\right) n^{2} Y^{2}+4 r(2 n r+r-3)-6 n r(3 n+2 r+8) Y\right] \\
& -\frac{r(n Y+2)}{3 n}\left[4 r(2 n+7)+3 n^{2}(2 n-2 r+19) Y^{2}+2 n((n-r)(r-6)\right. \\
& +(3 n-35 r-30)) Y], \\
g_{2}(Y) & =Y\left[-((n-r)(2 n-2 r+11)+32) n^{2} Y^{2}-4 r((n-r)(2 n+1)-4(n-1))\right. \\
& +6 n((n-r)(n+2 r+8)+8 r) Y]+\frac{(n(n-r+2) Y-2 r)}{3 n}[4 r(2 n+7) \\
& \left.+3 n^{2}(2 n-2 r+19) Y^{2}+2 n((n-r)(r-6)+(3 n-35 r-30)) Y\right] .
\end{aligned}
$$

and without having solve to (3.122) explicitly, even in the case of the existence of a real solution, $H$ will be proportional to $\lambda$ with a numerical factor $\nu$, where $\nu$ be the root of the equation (3.122). Hence, we can assume that $H=\nu \lambda$ and substituting it in (3.107) and (3.108), and using (3.96), (3.97) and (3.98), we obtain

$$
\begin{gathered}
-\lambda e_{n} e_{n}(\lambda)+\frac{e_{n}^{2}(\lambda)(n \nu+4)}{n \nu+2}=\frac{n \nu(n \nu+2) \lambda^{4}}{4}, \\
e_{n}^{2}(\lambda)=-\frac{(n \nu+2)(n(n-r+2) \nu-2 r) \lambda^{4}}{4(n-r-1)}, \\
\lambda e_{n} e_{n}(\lambda)-e_{n}^{2}(\lambda)\left(1+\frac{3 \nu n-2 r}{n(n-r+2) \nu-2 r}\right)=-\frac{n \nu(n(n-r+2) \nu-2 r) \lambda^{4}}{4(n-r-1)} .
\end{gathered}
$$

Adding (3.123) and (3.125), we find

$$
e_{n}^{2}(\lambda)=\frac{(n \nu+2)(n(n-r+2) \nu-2 r) \lambda^{4}}{4(n-r-1)} .
$$


Using (3.124) and (3.126), we get $e_{n}(\lambda)=0$. Since $H=\nu \lambda$, therefore we obtain $e_{n}(H)=0$, a contradiction to (3.65). Which completes the proof of Proposition 3.6.

Now, we consider the case of two distinct eigenvalues.

Case IV: Let either of $\lambda-\lambda_{1}=0$ or $\lambda_{n}-\lambda_{1}=0$ or $\lambda-\lambda_{n}=0$. Then, from (3.64), we can say that each eigen value $\lambda, \lambda_{1}$ and $\lambda_{n}$ is the multiple of $H$. From (3.65), we have

$$
e_{a}(\lambda)=e_{a}\left(\lambda_{1}\right)=e_{a}\left(\lambda_{n}\right)=0, \quad \text { for } \quad a=1,2, \ldots, n-1 .
$$

If $\lambda-\lambda_{n}=0$ or $\lambda_{n}-\lambda_{1}=0$, then from (3.33) or (3.39), we get $e_{n}(H)=0$ which is a contradiction to (3.65). Now, if $\lambda-\lambda_{1}=0$, then $r=n-1$. From (3.64), we have

$$
\lambda=\lambda_{1}=\frac{3 n H}{2(n-1)} .
$$

Putting $r=n-1$ in (3.81) and using (3.128), we get

$$
-(n-1) \omega_{12}^{n} e_{n}(H)-e_{n} e_{n}(H)+\frac{n^{2}(n+8)}{4(n-1)} H^{3}=0 .
$$

Using (3.128) in (3.96), we find

$$
e_{n}\left(\omega_{12}^{n}\right)+\left(\omega_{12}^{n}\right)^{2}=\frac{3 n^{2} H^{2}}{4(n-1)} .
$$

Using (3.5), (3.64) and (3.128) in (3.12), we have

$$
e_{n}(H)=-\frac{n+2}{3} H \omega_{12}^{n} .
$$

Differentiating (3.131) along $e_{n}$ and using (3.125), we get

$$
e_{n} e_{n}(H)=\frac{(n+2)(n+5)}{9} H\left(\omega_{12}^{n}\right)^{2}-\frac{n^{2}(n+2)}{4(n-1)} H^{3} .
$$

Eliminating $e_{n} e_{n}(H)$ from (3.129) and (3.132), we obtain

$$
\frac{2(n+2)(n-4)}{9}\left(\omega_{12}^{n}\right)^{2}+\frac{n^{2}(n+5)}{2(n-1)} H^{2}=0 .
$$

Differentiating, again (3.133) along $e_{n}$ and using (3.130) and (3.131), we get

$$
\frac{4(n-4)}{9}\left(\omega_{12}^{n}\right)^{2}+\frac{3 n^{2}}{(n-1)} H^{2}=0 .
$$

Therefore, from (3.133) and (3.134), we can conclude that $H$ must be zero.

Combining Proposition 3.6 and Case IV, we have

Proposition 3.3. Let $M_{1}^{n}$ be a biharmonic Lorentz hypersurface in the pseudo Euclidean space $E_{1}^{n+1}$, having the nondiagonal shape operator given by (2.11). If gradH is space like, then $M_{1}^{n}$ is not proper biharmonic.

Now, using Propositions 3.2 and 3.7, we have following:

Theorem 3.1. Let $M_{1}^{n}$ be a biharmonic Lorentz hypersurface in the pseudo Euclidean space $E_{1}^{n+1}$, having non-diagonal shape operator given by (2.11) with at most three distinct principal curvatures. Then $M_{1}^{n}$ is not proper biharmonic.

\section{Acknowledgments}

The first author is grateful to Guru Gobind Singh Indraprastha University for providing IPRF fellowship to pursue research. 


\section{References}

[1] Arvanitoyeorgos, A., Defever, F. and Kaimakamis, G., Hypersurfaces of $E_{s}^{4}$ with proper mean curvature vector. J. Math. Soc. Japan, 59 (2007), 3, 797-809.

[2] Arvanitoyeorgos, A., Defever, F., Kaimakamis, G. and Papantoniou, V., Biharmonic Lorentzian hypersurfaces in E. $E_{1}^{4}$ Pac. J. Math. 229 (2007), 2, 293-305.

[3] Chen, B. Y., Total Mean Curvature and Submanifolds of Finite Type. World Scientific, Singapore, 1984.

[4] Chen, B. Y., Submanifolds of finite type and applications. Proc. Geometry and Topology Research Center, Taegu, 3 (1993), 1-48.

[5] Chen, B. Y., A report on submanifolds of finite type. Soochow J. Math., 22 (1996); 22: 117-337.

[6] Chen, B. Y., Classification of marginally trapped Lorentzian flat surfaces in $E_{1}^{4}$ and its application to biharmonic surfaces. J. Math. Anal. Appl., 340(2008), 861-875.

[7] Chen, B. Y. and Ishikawa, S., Biharmonic surfaces in pseudo-Euclidean spaces. Mem. Fac. Sci. Kyushu Univ. A., 45 (1991), $323-347$.

[8] Chen, B. Y. and Ishikawa, S., Biharmonic pseudo-Riemannian submanifolds in pseudo-Euclidean spaces. Kyushu J. Math., 52 (1998), 1-18.

[9] Dimitric, I., Quadratic representation and submanifolds of finite type. Doctoral thesis, Michigan State University, 1989.

[10] Deepika and Gupta, R. S., Biharmonic hypersurfaces in $E^{5}$ with zero scalar curvature. Afr. Diaspora J. Math., 18 (2015), 1, $12-26$.

[11] Fu, Y., Biharmonic hypersurfaces with three distinct principal curvatures in the Euclidean 5-space, Journal of Geometry and Physics, 75 (2014), 113-119.

[12] Gupta, R. S., On biharmonic hypersurfaces in Euclidean space of arbitrary dimension. Glasgow Math. J., 57 (2015), $633-642$.

[13] Gupta, R. S., Biharmonic hypersurfaces in $E_{s}^{5}$. An. Stiint. Univ. Al. I. Cuza Iasi Mat. (N.S.), Tomul LXII (2016), f. 2, vol. 2 , 585-593.

[14] Hasanis, Th. and Vlachos, Th., Hypersurfaces in $E^{4}$ with harmonic mean curvature vector field. Math. Nachr., 172 (1995), 145-169.

[15] Magid, M. A., Lorentzian isoparametric hypersurfaces. Pacific J. Math., 118(1985), 165-197.

[16] Petrov, A. Z., Einstein spaces. Pergamon Press, Oxford, 1969.

\section{Affiliations}

\section{DEEPIKA}

ADDRESS: University School of Basic and Applied Sciences,

Guru Gobind Singh Indraprastha University,

Sector-16C, Dwarka, New Delhi-110078, India.

E-MAIL: sdeep2007@gmail.com

RAM SHANKAR GUPTA

AdDress: Assistant Professor,

University School of Basic and Applied Sciences,

Guru Gobind Singh Indraprastha University,

Sector-16C, Dwarka, New Delhi-110078, India.

E-MAIL: ramshankar.gupta@gmail.com 